\title{
LATEST PROGRESS OF FAULT DETECTION AND LOCALIZATION IN COMPLEX ELECTRICAL ENGINEERING
}

\author{
Zheng Zhao - Can Wang — Yagang Zhang — Yi Sun *
}

\begin{abstract}
In the researches of complex electrical engineering, efficient fault detection and localization schemes are essential to quickly detect and locate faults so that appropriate and timely corrective mitigating and maintenance actions can be taken. In this paper, under the current measurement precision of PMU, we will put forward a new type of fault detection and localization technology based on fault factor feature extraction. Lots of simulating experiments indicate that, although there are disturbances of white Gaussian stochastic noise, based on fault factor feature extraction principal, the fault detection and localization results are still accurate and reliable, which also identifies that the fault detection and localization technology has strong anti-interference ability and great redundancy.
\end{abstract}

Keywords: fault detection and localization, feature extraction, WAMS, PMU, EMS

\section{INTRODUCTION}

With the rapid development of power generation industry in China, the scale of power grid has expanded rapidly. Meanwhile, owing to the large scale of the gridconnected renewable power plants such as solar power, wind power and etc, the implementation and realization of the west-to-east power transmission, modern power structure is becoming more and more complex. With the increasing scale and complexity of power system, the probability of system fault is also increasing in a geometric progression. The significant economic losses and social consequences will take place if it can't receive timely and effective treatment when abnormal condition of the devices is happened, so the control and protection system for power system is facing unprecedented challenges [1-3].

In order to guarantee the reliability and safety of a complex power system, efficient fault detection and localization (FDL) schemes are essential to quickly detect and locate faults so that appropriate and timely corrective mitigating and maintenance actions can be taken. FDL methods can be classified into three major categories: model-based, knowledge-based and data-driven approaches [4-6]. Since faults in complex system are hierarchical, correlation, time delay and uncertainty, the process is too complex to be modeled analytically and it is the lack of expert knowledge. The data-driven method is supposed not to require an explicit or complete model of the complex system, so it is preferred when system monitoring data for the nominal and degraded conditions is available. At present, complex power system has established a complete Supervisory Control and Data Acquisition (SCADA) system for primary and auxiliary equipments [7-10], which can provide reliable real-time data for fault detection and localization. On the other hand, as these model-based, knowledge-based and data-driven FDL methods have their pros and cons, it is a trend that these three complementary techniques are usually integrated together to achieve a better performance for complex system [11].

According to complex power systems, we have carried out large numbers of basic researches. In paper [12], in order to meet the requirements of wide area intelligent control, this paper puts forward a new fault location scheme based on Bayesian discriminant analysis theory. And BDA fault detection is proposed to give a partition for the membership of each element (healthy or faulted), in which the node status quantities are adopted as basic data provide by PMU. Paper [13] used mainly pattern classification technology and linear discrimination principle of pattern recognition theory to search for laws of electrical quantity marked changes. In paper [14], PCA theory is introduced into the field of fault detection to locate precisely the fault by mean of the voltage and current phasor data from the PMUs. Massive simulation experiments have fully proven that the fault identification can be performed successfully by PCA and calculation. In this paper, under the current measurement precision of PMU, we will put forward a new type of fault detection and localization technology based on fault factor feature extraction.

The paper is organized as follows. In Section 2, the theoretical basis of fault detection and localization is introduced. In Section 3, considering the current measurement precision of PMUs, according to fault factor feature extraction theory, the fault detection and localization in complex electric power systems is clarified in detail. Finally, the paper is concluded in Section 4.

\section{THEORETICAL BASIS OF FAULT DETECTION AND LOCALIZATION}

Contemporary science and technology has generated and is generating so vast amounts of data at unprecedented rate and scale, in order to classify these data, fea-

\footnotetext{
* Department of Electrical Engineering, North China Electric Power University, Box 205, Baoding, Hebei 071003, PR China. yagangzhang@gmail.com
} 


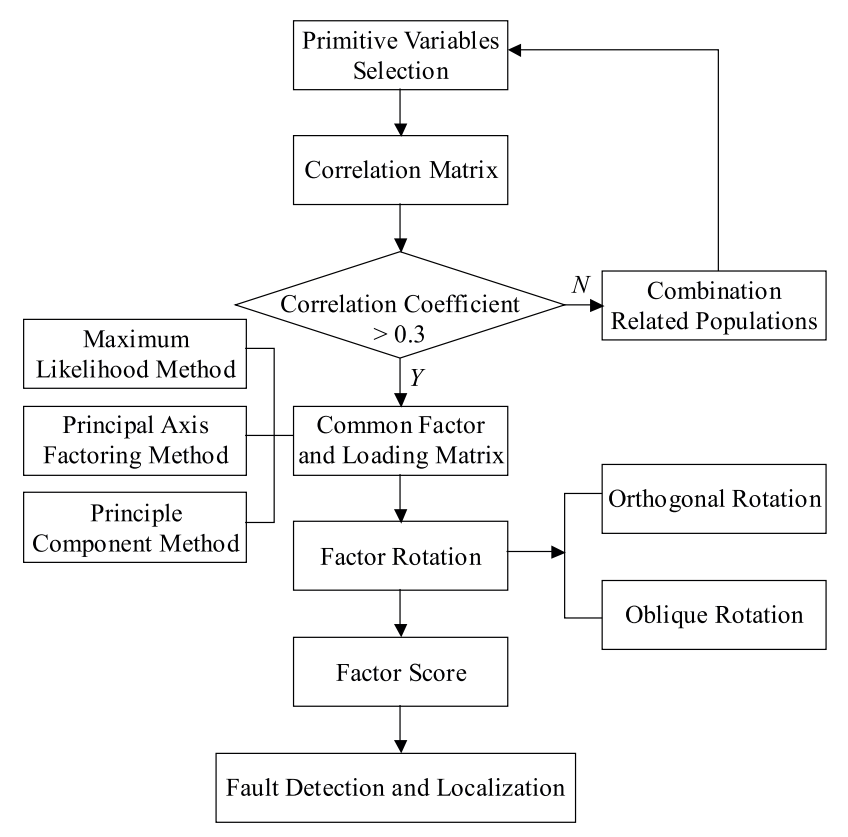

Fig. 1. Fault detection and localization algorithm flow

ture extraction has become ubiquitous. The purpose of feature extraction is to reduce the number of features of patterns and at the same time retain as much as possible of their discriminatory information $[15,16]$. For this reason, a good feature extractor chooses features which are similar for patterns in the same class and very different for patterns in different classes. Now let's introduce the general process of fault detection and localization based on fault factor feature extraction $[17,18]$.

For factor model

$$
\begin{gathered}
X_{i}=a_{i 1} F_{1}+a_{i 2} F_{2}+\cdots+a_{i m} F_{m}+\varepsilon_{i} \\
(i=1,2, \ldots, p)
\end{gathered}
$$

we can obtain, the covariance of $X_{i}$ and $F_{j}$ is

$$
\begin{aligned}
\operatorname{cov}\left(X_{i}, F_{j}\right)= & \operatorname{cov}\left[\sum_{k=1}^{m} a_{i k} F_{k}+\varepsilon_{i}, F_{j}\right]= \\
& \operatorname{cov}\left[\sum_{k=1}^{m} a_{i k} F_{k}, F_{j}\right]+\operatorname{cov}\left(\varepsilon_{i}, F_{j}\right)=a_{i j} .
\end{aligned}
$$

Suppose the factor loading matrix is $A$, the sum of squares of the $i$-th row's elements is

$$
h_{i}^{2}=\sum_{j=1}^{m} a_{i j}^{2} \quad(i=1,2, \ldots, p),
$$

which is just the communality of variable $X_{i}$.

From the factor model, we can know,

$$
\begin{aligned}
D\left(X_{i}\right)= & a_{i 1}^{2} D\left(F_{1}\right)+a_{i 2}^{2} D\left(F_{2}\right)+\cdots+a_{i m}^{2} D\left(F_{m}\right)+D\left(\varepsilon_{i}\right) \\
& =a_{i 1}^{2}+a_{i 2}^{2}+\cdots+a_{i m}^{2}+D\left(\varepsilon_{i}\right)=h_{i}^{2}+\sigma_{i}^{2} .
\end{aligned}
$$

The sum of squares of the $j$-th column's elements is

$$
g_{j}^{2}=\sum_{i=1}^{p} a_{i j}^{2} \quad(j=1,2, \ldots, m) .
$$

As we know, the solution of $A$ is not unique, here the solution will make the contribution $g_{1}^{2}=\sum_{i=1}^{p} a_{i 1}^{2}$ of the first common factor $F_{1}$ to $X$ reach the maximum, the contribution $g_{2}^{2}=\sum_{i=1}^{p} a_{i 2}^{2}$ of the second common factor $F_{2}$ to $X$ take second place, .., and the contribution of the $m$-th common factor $F_{m}$ to $X$ is the minimum.

The corresponding contributions are in sequence of:

$$
g_{1}^{2} \geq g_{2}^{2} \geq \cdots \geq g_{m}^{2}
$$

So, one can get

$$
\begin{gathered}
\sum_{j=1}^{p} \lambda_{i j} a_{j t}-\delta_{1 t} a_{i 1}=0, \\
(i=1,2, \ldots, p ; \quad t=1,2, \ldots, m) \\
\delta_{1 t}= \begin{cases}1, & t=1, \\
0, & t \neq 1 .\end{cases}
\end{gathered}
$$

Left multiplication $a_{i 1}$ and sum $i$, then

$$
\begin{gathered}
\sum_{j=1}^{p}\left[\sum_{i=1}^{p} \lambda_{i j} a_{i 1}\right] a_{j t}-\delta_{1 t} \sum_{i=1}^{p} a_{i 1}^{2}=0 \\
(t=1,2, \ldots, m)
\end{gathered}
$$

Here we should take notice of

$$
\begin{array}{r}
g_{1}^{2}=\sum_{i=1}^{p} a_{i 1}^{2}, \quad \sum_{i=1}^{p} \lambda_{i j} a_{i 1}=\sum_{i=1}^{p} \lambda_{j i} a_{i 1}=a_{j i} . \\
\sum_{j=1}^{p} a_{j 1} a_{j t}-\delta_{1 t} g_{1}^{2}=0 \quad(t=1,2, \ldots, m) .
\end{array}
$$

Similarly, left multiplication $a_{i t}$ with the former formula and sum $t$, then

$$
\begin{gathered}
\sum_{j=1}^{p} a_{j 1}\left[\sum_{t=1}^{m} a_{j t} a_{i t}\right]-\sum_{t=1}^{m} \delta_{1 t} a_{i t} g_{1}^{2}=0 \\
(i=1,2, \ldots, p) .
\end{gathered}
$$

Then, for $r_{i j}^{*}=\sum_{t=1}^{m} a_{i t} a_{j t}$,

$$
\sum_{j=1}^{p} r_{i j}^{*} a_{j 1}=a_{i 1} g_{1}^{2}, \quad(i=1,2, \ldots, p) .
$$

Or expressed as vectors,

$$
\begin{gathered}
\left(r_{i 1}^{*}, r_{i 2}^{*}, \ldots, r_{i p}^{*}\right)\left(\begin{array}{c}
a_{11} \\
\vdots \\
a_{p 1}
\end{array}\right)=a_{i 1} g_{1}^{2} \\
(i=1,2, \ldots, p) .
\end{gathered}
$$




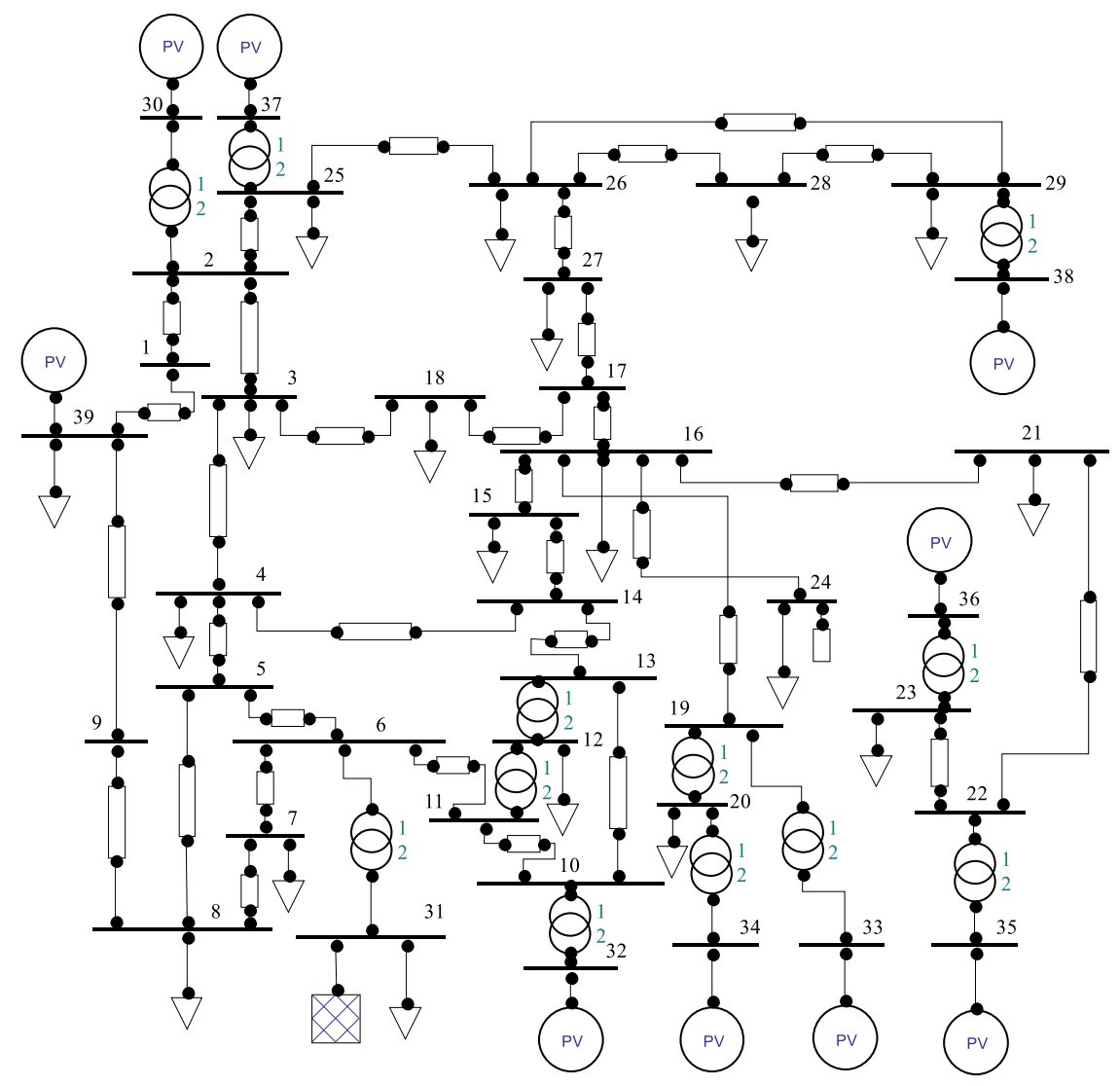

Fig. 2. Electric diagram of IEEE 39-node system

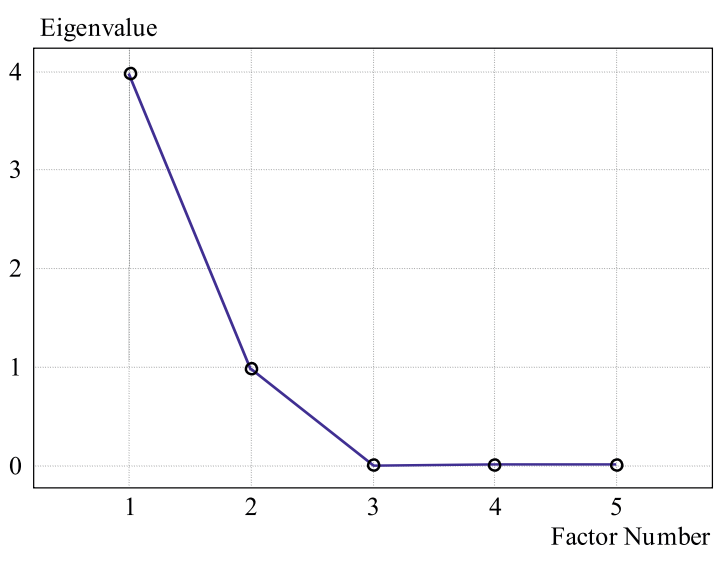

Fig. 3. Screen plot of all eigenvalues

$$
\left(R^{*}-I g_{1}^{2}\right) A_{1}=0 .
$$

Therefore $g_{1}^{2}$ is the biggest eigenvalue of approximate correlation matrix $R^{*}$, and $A_{1}$ is the eigenvector that corresponds to $g_{1}^{2}$.

Obviously, $A_{1}$ is still an eigenvector that corresponds to $\lambda_{1}^{*}$, and satisfies $A_{1}^{\prime} A_{1}=\lambda_{1}^{*} t_{1}^{* \prime} t_{1}^{*}=\lambda_{1}^{*}=g_{1}^{2}$.

In order to obtain the rest of $m-1$ columns in the factor loading matrix $A$, we should introduce the decomposition formula of spectrum of $R^{*}$,

$$
R^{*}=\sum_{i=1}^{p} \lambda_{i}^{*} t_{i}^{*} t_{i}^{* \prime}=A_{1} A_{1}^{\prime}+\sum_{i=2}^{p} \lambda_{i}^{*} t_{i}^{*} t_{i}^{* \prime} .
$$

Particularly, $R^{*}$ can also be decomposed into

$$
R^{*}=A A^{\prime}=\left(A_{1}, \ldots, A_{m}\right)\left(\begin{array}{c}
A_{1}^{\prime} \\
\vdots \\
A_{m}^{\prime}
\end{array}\right)=\sum_{t=1}^{m} A_{t} A_{t}^{\prime} .
$$

That is to say, after $A_{1}$ is obtained, subtract $A_{1} A_{1}^{\prime}$ from $R^{*}$, one can get

$$
R^{*}-A_{1} A_{1}^{\prime}=\sum_{t=2}^{m} A_{t} A_{t}^{\prime} .
$$

In this way, one has solved the factor loading matrix $A$ from $R^{*}$. Combining the researches in this paper, we have put forward the following fault detection and localization algorithm flow, see Fig. 1.

\section{FAULT DETECTION AND LOCALIZATION IN COMPLEX POWER SYSTEM BASED ON FAULT FACTOR FEATURE EXTRACTION}

In order to illustrate the specific procedure of fault detection and localization, let us take IEEE 39-node system as example. The electric diagram of IEEE 39-node system has been present in Fig. 2. In the power network structure, Node-18 appears single-phase short circuit fault. By BPA simulations, utilizing the actual measurement information of corresponding node negative sequence voltages, we will carry out fault detection and localization of fault component and non-fault component. 
Table 1. The white Gaussian stochastic noise $N\left(0,0.005^{2}\right)$

\begin{tabular}{rrrrrr}
\hline No. & 1 & \multicolumn{1}{c}{2} & 3 & 4 & \multicolumn{1}{c}{5} \\
\hline 1 & 0.0065 & 0.0078 & -0.0080 & -0.0029 & 0.0071 \\
2 & 0.0012 & 0.0130 & -0.0057 & -0.0116 & 0.0047 \\
3 & -0.0013 & 0.0019 & 0.0023 & 0.0009 & -0.0062 \\
4 & 0.0009 & 0.0081 & 0.0012 & -0.0017 & 0.0012 \\
5 & 0.0019 & -0.0022 & 0.0039 & -0.0053 & 0.0059 \\
6 & 0.0062 & -0.0075 & -0.0087 & 0.0011 & -0.0077 \\
7 & -0.0008 & -0.0050 & -0.0017 & -0.0044 & 0.0017 \\
8 & 0.0007 & 0.0045 & -0.0072 & 0.0023 & -0.0004 \\
9 & -0.0006 & -0.0131 & -0.0068 & 0.0072 & 0.0021 \\
10 & -0.0005 & 0.0001 & -0.0059 & -0.0019 & -0.0070 \\
11 & -0.0059 & -0.0027 & -0.0004 & -0.0041 & -0.0088 \\
12 & -0.0015 & 0.0042 & 0.0065 & 0.0062 & -0.0036 \\
13 & 0.0032 & 0.0048 & -0.0031 & 0.0033 & -0.0057 \\
14 & -0.0010 & -0.0031 & -0.0018 & 0.0007 & -0.0014 \\
15 & 0.0023 & -0.0046 & -0.0035 & 0.0027 & 0.0002 \\
16 & 0.0040 & -0.0072 & 0.0013 & 0.0038 & -0.0028 \\
17 & 0.0063 & 0.0029 & 0.0060 & -0.0008 & 0.0060 \\
18 & -0.0037 & 0.0051 & -0.0067 & 0.0005 & -0.0001 \\
19 & 0.0028 & -0.0056 & 0.0003 & 0.0016 & -0.0106 \\
20 & 0.0056 & -0.0078 & -0.0031 & 0.0050 & 0.0059 \\
21 & 0.0102 & -0.0066 & -0.0051 & -0.0013 & -0.0099 \\
22 & 0.0027 & 0.0030 & 0.0009 & 0.0042 & -0.0007 \\
23 & -0.0050 & -0.0074 & -0.0028 & 0.0077 & -0.0041 \\
24 & 0.0032 & 0.0030 & -0.0082 & 0.0057 & 0.0061 \\
25 & -0.0071 & 0.0021 & -0.0013 & -0.0025 & -0.0030 \\
26 & 0.0017 & 0.0066 & 0.0011 & 0.0048 & 0.0079 \\
27 & -0.0044 & 0.0072 & -0.0009 & 0.0009 & -0.0032 \\
28 & 0.0033 & -0.0010 & 0.0011 & -0.0020 & -0.0033 \\
29 & 0.0035 & 0.0056 & 0.0036 & 0.0059 & 0.0000 \\
30 & 0.0083 & 0.0009 & -0.0013 & 0.0097 & -0.0024 \\
31 & 0.0039 & -0.0052 & 0.0022 & -0.0005 & 0.0022 \\
32 & 0.0000 & 0.0008 & -0.0031 & -0.0007 & 0.0047 \\
33 & -0.0056 & -0.0008 & 0.0057 & 0.0115 & 0.0009 \\
34 & -0.0048 & -0.0100 & 0.0053 & 0.0052 & 0.0019 \\
35 & 0.0045 & -0.0012 & -0.0024 & 0.0009 & -0.0058 \\
36 & -0.0022 & -0.0014 & -0.0028 & 0.0035 & 0.0014 \\
37 & -0.0054 & -0.0105 & 0.0025 & 0.0077 & -0.0034 \\
38 & 0.0002 & 0.0067 & -0.0165 & 0.0045 & 0.0001 \\
39 & 0.0018 & -0.0022 & 0.0032 & 0.0075 & -0.0092 \\
\hline & & & & &
\end{tabular}

Table 2. The factor score based on fault factor feature extraction

\begin{tabular}{crrrr}
\hline Node & Factor 1 & Factor 2 & Factor 3 & Factor 4 \\
\hline Node-1 & -0.44395 & -0.49280 & 0.87553 & 0.24133 \\
Node-2 & 0.57297 & -0.04003 & -1.22361 & -1.19348 \\
Node-3 & 1.40257 & 1.11664 & 0.38385 & 0.37991 \\
Node-4 & 0.06630 & -0.21294 & -0.09277 & -1.03919 \\
Node-5 & -0.46603 & 0.38499 & -0.00612 & 0.50921 \\
Node-6 & -0.26291 & -0.54509 & 1.28537 & 0.42549 \\
Node-7 & -0.60332 & -0.18063 & -0.38719 & -0.44588 \\
Node-8 & -0.33602 & -1.62879 & 0.31398 & 0.35989 \\
Node-9 & -0.65318 & -1.35568 & -0.38614 & -1.13082 \\
Node-10 & -0.39315 & 0.10149 & 0.86692 & 1.20142 \\
Node-11 & -0.70409 & 1.62940 & 0.18193 & 0.11525 \\
Node-12 & -0.26625 & -0.61454 & -0.17091 & 0.42017 \\
Node-13 & -0.34271 & 0.38639 & 0.24177 & -0.02006 \\
Node-14 & -0.06004 & -0.21223 & 0.16999 & -0.08811 \\
Node-15 & 0.46920 & -0.61847 & -0.22849 & -0.16308 \\
Node-16 & 0.68312 & 0.42142 & -0.42142 & -0.79325 \\
Node-17 & 2.26504 & 0.53895 & 0.56081 & 0.76377 \\
Node-18 & 4.27460 & -0.36171 & -0.14535 & 0.21392 \\
Node-19 & -0.56137 & 1.81217 & 0.77326 & -0.32941 \\
Node-20 & -0.74520 & 0.34073 & 0.00268 & -0.50687 \\
Node-21 & 0.12791 & 0.86519 & 1.05526 & 0.21332 \\
Node-22 & -0.22920 & -0.02865 & -0.57716 & -0.23579 \\
Node-23 & -0.17407 & 0.30401 & -0.74267 & -0.58871 \\
Node-24 & 0.78669 & -0.14123 & 0.12440 & 0.98113 \\
Node-25 & 0.09824 & 0.44440 & -0.09947 & 0.12809 \\
Node-26 & 0.55588 & 1.50461 & -0.13367 & -0.54253 \\
Node-27 & 1.27936 & 0.62832 & 0.00489 & -0.80362 \\
Node-28 & -0.02434 & 0.48766 & 0.03051 & 0.26376 \\
Node-29 & 0.05982 & -0.67112 & -0.75002 & -0.34172 \\
Node-30 & -0.59569 & 0.50746 & -1.33860 & 0.25895 \\
Node-31 & -0.83349 & -0.40088 & 0.42127 & -0.32255 \\
Node-32 & -0.86409 & 0.02037 & 0.70302 & 0.60860 \\
Node-33 & -0.54070 & -0.66954 & -1.40588 & 0.77547 \\
Node-34 & -0.62377 & -1.00744 & -0.20210 & -0.75602 \\
Node-35 & -0.69769 & 0.48405 & 0.66287 & 0.53551 \\
Node-36 & -0.73154 & -0.41790 & -0.14615 & 0.32858 \\
Node-37 & -0.37451 & 0.07010 & -0.79960 & -1.18108 \\
Node-38 & 0.29366 & -3.48841 & 0.62058 & 1.22821 \\
Node-39 & -1.40804 & 1.03975 & -0.02157 & 0.53016 \\
\hline & & & &
\end{tabular}

Table 3. The factor score based on fault factor feature extraction

\begin{tabular}{|c|c|c|c|c|c|c|c|c|c|}
\hline \multirow{2}{*}{ Factor } & \multicolumn{3}{|c|}{ Initial Eigenvalues } & \multicolumn{3}{|c|}{$\begin{array}{c}\text { Extraction Sums of Squared } \\
\text { Loadings }\end{array}$} & \multicolumn{3}{|c|}{$\begin{array}{c}\text { Rotation Sums of Squared } \\
\text { Loadings }\end{array}$} \\
\hline & Total & $\begin{array}{c}\% \text { of } \\
\text { Variance }\end{array}$ & $\underset{\%}{\text { Cumulative }}$ & Total & $\begin{array}{c}\% \text { of } \\
\text { Variance }\end{array}$ & $\underset{\%}{\text { Cumulative }}$ & Total & $\begin{array}{c}\% \text { of } \\
\text { Variance }\end{array}$ & $\underset{\%}{\text { Cumulative }}$ \\
\hline 1 & 3.974 & 79.483 & 79.483 & 3.965 & 79.306 & 79.306 & 3.835 & 76.694 & 76.694 \\
\hline 2 & 0.988 & 19.758 & 99.241 & 0.090 & 1.792 & 81.098 & 0.220 & 4.404 & 81.097 \\
\hline 3 & 0.016 & 0.320 & 99.561 & 0.008 & 0.158 & 81.256 & 0.005 & 0.104 & 81.201 \\
\hline 4 & 0.012 & 0.236 & 99.797 & 0.002 & 0.047 & 81.303 & 0.005 & 0.102 & 81.303 \\
\hline 5 & 0.010 & 0.203 & 100.000 & & & & & & \\
\hline
\end{tabular}

According to the current measurement precision of PMU, suppose the standard deviation of voltage vector is 0.005 , and the mean error is 0 [19]. Let us introduce a white Gaussian stochastic noise $N\left(0,0.005^{2}\right)$, the influence on IEEE 39-node system is present in Table 1.

By the principal of fault factor feature extraction, the correlation matrix and the communalities of variables have been calculated. Based on this, one has obtained initial eigenvalues, see Table 3. In this place, the feature extraction method is principal axis factoring. In the total variance explained, the eigenvalue of the first factor is 3.974 , the proportion of variance is $79.483 \%$, of course, the cumulative proportion of variance is also $79.483 \%$ (close to $80 \%$ ). The eigenvalue of the second factor is 0.988 , the proportion of variance is $19.758 \%$, and the corresponding cumulative proportion of variance is $99.241 \%$ (close to $100 \%$ ). The eigenvalue of the third factor is 0.016 , the proportion of variance is $0.320 \%$ (it is very small), and the corresponding cumulative proportion of variance is $99.561 \%$, and so on. Figure 3 is the screen plot of these eigenvalues.

Likewise, using principal axis factoring feature extraction method, one can further solve factor matrix, rotated factor matrix and factor score coefficient matrix. Finally, 
the factor score results based on fault factor feature extraction are obtained, see Table 2.

According to the ultimate factor score results, the fault feature is obvious. In the anterior studies, the first factor corresponding to the cumulative proportion of variance is $79.483 \%$, the eigenvalue is 3.974 . So, the first factor is our focus. At the same time, let us pay attention to the factor score of factor 1 in Table 2, there are 39 nodes altogether, and the score of Node- 18 is 4.27460 , which is the biggest one. As we have known, the Node-18 is just the actual fault component.

In these simulations, although there are disturbances of white Gaussian stochastic noise, based on fault factor feature extraction principal, the fault detection and localization results are still accurate and reliable, which also indicates that the fault detection and localization based on fault factor feature extraction principal has strong anti-interference ability and great redundancy.

\section{CONCLUSIONS}

Feature extraction for classification achieves this dimensionality reduction by maximizing a suitably chosen objective function, thus preserving or enhancing the class separability in the feature domain. Efficient feature extraction and classification techniques are essential for analysis of multivariate statistical data $[15,18]$.

The new type of smart grid can utilize different kinds of information in a larger scale. In the researches of complex electrical engineering, efficient fault detection and localization schemes are essential to quickly detect and locate faults so that appropriate and timely corrective mitigating and maintenance actions can be taken. In this paper, under the current measurement precision of PMU, we have put forward a new type of fault detection and localization technology based on fault factor feature extraction. The results of massive simulations have confirmed, although there are disturbances of white Gaussian stochastic noise, based on fault factor feature extraction principal, the fault detection and localization results are still accurate and reliable. The researches have also identified the strong anti-interference ability and great redundancy of the fault detection and localization technology presented in this paper.

\section{Acknowledgements}

This research was supported partly by the National Key Basic Research Project (973 Program) of China (2012CB215200), the NSFC (51277193), the Specialized Research Fund for the Doctoral Program of Higher Education (20110036110003), the Fundamental Research Funds for the Central Universities (12MS120, 14ZD22) and the Natural Science Foundation of Hebei Province.

\section{REFERENCES}

[1] NASAR, A. A. S.-GORAN, K.-ALEKSANDRA, M.-ZELJKO, D.: Sensor Fault Detection and Isolation in a Thermal
Power Plant Steam Separator, Control Engineering Practice 21 (2013), 908.

[2] GARLAPATI, S.-LIN, H.-HEIER, A.-SHUKLA, S. K.THORP, J. : A Hierarchically Distributed Non-Intrusive Agent Aided Distance Relaying Protection Scheme to Supervise Zone 3, International Journal of Electrical Power \& Energy Systems 50 (2013), 42.

[3] RAMAN, S.-GOKARAJU, R.-JAIN, A. : An Adaptive Fuzzy MHS Relay for Phase Backup Protection with Infeed from STATCOM, IEEE Transactions on Power Delivery 28 (2013), 120.

[4] ALI, A.-MAHDI, D. : Data Driven Approach for Fault Detection and Diagnosis of Turbine in Thermal Power Plant using Independent Component Analysis (ICA), International Journal of Electrical Power \& Energy System 43 (2013), 728.

[5] HARROU, F.-NOUNOU, M. N.-NOUNOU, H. N.-MADAKYARU, M.: Statistical Fault Detection using PCA-Based GLR Hypothesis Testing, Journal of Loss Prevention in the Process Industries 26 (2013), 129..

[6] GRBOVIC, M.-LI, W. C.-XU, P.-USADI, A. K.-SONG, L. M.-VUCETIC, S.: Decentralized Fault Detection and Diagnosis via Sparse PCA based Decomposition and Maximum Entropy Decision Fusion, Journal of Process Control 22 (2012), 738.

[7] YANG, Z. J.-LING, B. W. K.-BINGHAM, C.: Fault Detection and Signal Reconstruction for Increasing Operational Availability of Industrial Gas Turbines, Measurement 46 (2013), 1938.

[8] ZHANG, Y. G.-WANG, Z. P.-ZHANG, J. F.: Fault Identification based on NLPCA in Complex Electrical Engineering, Journal of Electrical Engineering 63 (2012), 255.

[9] MACHOWSKI, J.-KACEJKO, P.-NOGAL,.-WANCERZ, M.: Power System Stability Enhancement by WAMS-Based Supplementary Control of Multi-Terminal HVDC Networks, Control Engineering Practice 21 (2013), 583.

10] FARROKH, A.-MAHMUD, F. F.-AMIR, S. : Optimal PMU Placement based on Probabilistic Cost/Benefit Analysis, IEEE Transactions on Power Systems 28 (2013), 566.

[11] DAI, X. W.-GAO, Z. W. : From Model, Signal to Knowledge: a Data-Driven Perspective of Fault Detection and Diagnosis, IEEE Transactions on Industrial Informatics 9 (2013), in press.

[12] ZHANG, Y. G.-WANG, Z. P.-ZHAO, S. Q.: BDA Fault Detection in Complex Electric Power Systems, International Review of Electrical Engineering 7 (2012), 3638.

[13] ZHANG, Y. G.-WANG, Z. P.-ZHANG, J. F.-MA, J. : Fault Localization in Electrical Power Systems: A Pattern Recognition Approach, International Journal of Electrical Power \& Energy Systems 33 (2011), 791

14] ZHANG, Y. G.-WANG, Z. P.-ZHANG, J. F.: A Novel Fault Identification using WAMS/PMUjour Advances in Electrical and Computer Engineering vol12.

[15] HAKAN, C. : Feature extraction techniques in high-dimensional spaces: Linear and nonlinear approaches, $\mathrm{PhD}$ thesis of Vanderbilt University, 2005.

[16] ZHU, J. P.: Applied Multivariate Statistical Analysis, Science Press, Beijing, 2006.

[17] YANG, Y. Y.-LU, J. M.-ROVNAK, J.-QUACKENBUSH, S. L.-LUNDQUIST, E. A.: SWAN-1, a Caenorhabditis Elegans WD Repeat Protein of the AN11 Family, is a Negative Regulator of rac GTPase Function, Genetics 174 (2006), 1917-1932.

[18] KOEL, D.: Feature Extraction and Classification for LargeScale Data Analysis, PhD thesis of California University, Irvine, 2007.

[19] IEEE STD C37.118 ${ }^{\mathrm{TM}}$-2005: IEEE Standard for Synchrophasors for Power Systems, IEEE, New York, 2006.

Received 26 April 2013 